\title{
Even promising Alzheimer's research has gaps
}

\author{
David Webber \\ is a biotechnol- \\ ogy analyst at \\ Alex Brown \& \\ Sons (New York).
}

Great progress has been made in understanding the mechanisms of Alzheimer's disease (AD). The level of knowledge is so much higher than it was a decade ago that several therapeutic approaches are being investigated. At the same time, there remains a lack of consensus on fundamental issues. For example, the two major physical manifestations in the brains of $\mathrm{AD}$ patients are amyloid plaques and neurofibrillary tangles. There is still no final consensus on whether plaques and tangles cause $\mathrm{AD}$ or result from $\mathrm{AD}$ or even, in the case of plaques, result from mere aging. Nor is there complete consensus on whether $\mathrm{AD}$ is a genetic disease or an environmental disease, originating in some insult to the body.

Development of drugs for AD must be considered early and risky. Among groups working on therapeutics that target actual disease mechanisms rather than symptoms, even leaders are the better part of a year away from selecting a lead compound. And since drug development will be running ahead of basic research, most developers will not learn whether they have chosen the correct therapeutic targets until they conduct clinical efficacy studies.

Still, in spite of the scientific gaps, AD programs at biotechnology companies should be viewed as important assets. A large and growing market, for a costly and devastating illness for which no therapies exist and for which several may be appropriate, represents an outstanding commercial as well as humanitarian opportunity.

$\mathrm{AD}$ is one of the most significant disorders, with an estimated cost to American society of $\$ 90$ billion per year. In the U.S., there are approximately 4 million cases of AD and 100,000 deaths per year attributable to $\mathrm{AD}$. Annually, home care costs $\$ 18,000$ per AD patient, while nursing-home charges come to $\$ 36,000$.

It is known that AD's amyloid plaques are comprised largely of aggregates of a peptide called betaamyloid and that AD's neurofibrillary tangles consist of fibers of the tau protein. Yet current diagnostic techniques, which can only be per- formed at autopsy, do not explain the relationship of these pathological features to age and disease state. In fact, the field has split into two camps. One camp believes that improper processing of beta amyloid leads to plaque formation, which is followed by tangles and cell death. Another camp believes that the improper processing of tau protein leads to tangles and that plaque formation is either a background event unrelated to AD's pathology or a consequence of tangle formation.

Current therapeutic strategies for $\mathrm{AD}$ revolve around decreasing the rate at which patients decline. While this may be scientifically and medically unsatisfying, small improvements in the rates of patient deterioration could nevertheless be the source of useful and approvable therapeutics.

In the course of $\mathrm{AD}$, cholinergic neurons that produce acetylcholine are lost in the brain. An obvious therapeutic approach, therefore, is to attempt to enhance the activity of the remaining acetylcholine. This can be achieved by inhibiting the enzyme that breaks down acetylcholine, by increasing the release of acetylcholine from the remaining cholinergic neurons, or by stimulating the receptor to which acetylcholine binds. Although cholinergic therapy has a rational basis for development, it has never been established that increasing the level of acetylcholine significantly affects the mental status of $\mathrm{AD}$ patients.

Beta amyloid also presents an appealing target for drug discovery. Though it is thought that a buildup of beta amyloid is toxic, the mechanisms of this toxicity are the subject of investigation. There is evidence that beta amyloid increases the level of calcium in cells, increases the toxicity of such excitatory neurotransmitters as glutamate, and increases the damage done by free radicals. Yet whether beta amyloid is toxic in its own right or is a potent cotoxin remains uncertain. A drug that reduces the level of beta amyloid produced by specific enzymes is the most obvious approach. Another approach involves limiting the damage done by beta amyloid.
Cephalon (W. Chester, PA) is developing nerve growth factor (NGF) to combat AD. Although AD patients have a normal amount of NGF, it is hoped that higher levels of the molecule might prevent the loss of cholinergic neurons. The therapeutic use of NGF itself would be difficult, however, since it doesn't cross the blood brain barrier. Consequently, Cephalon is looking for small molecules that can induce NGF production in relevant areas of the brain.

Regeneron Pharmaceuticals (Tarrytown, NY) is working on brain-derived neurotrophic growth factor (BDNF). BDNF is one of a family of neurotrophins, like NGF. These molecules promote neuronal survival during development, as well as the regeneration of damaged neurons. They act by binding to a family of tyrosine kinase receptors present in varying amounts on nerve cells.

While NGF has been a focus of interest because it seems to act specifically on cholinergic neurons, BDNF is believed to have a wider range of action based on the distribution of its tyrosine kinase receptor subtypes. In animal experiments, BDNF delivered directly to the cholinergic neurons resulted in an increase in cholinergic activity. Based on BDNF's receptor distribution, it should also have an effect on other types of neurons that are lost during the course of AD. Unlike NGF, BDNF appears to be downregulated in the AD brain, so insufficient amounts of BDNF may contribute to the pathology of the disease.

Cephalon is also developing calpain inhibitors for AD. Calpain is an enzyme that cleaves proteins, and excess amounts can lead to cell death. Calpain is induced in nerve cells by a high calcium concentration. High calcium concentrations can occur as a response to excitatory neurotransmitters such as glutamate. Glutamate release itself is a function of neuronal death. Cephalon is therefore working on small molecule inhibitors of calpain to block this hypothesized loop of neuronal destruction that may occur during the course of AD. III 